Annuaire suisse de politique de développement

Partenariats public-privé et coopération internationale

\title{
Privatisation de la guerre et sous-traitance des services publics : défis pour l'action humanitaire
}

Gilles Carbonnier

\section{OpenEdition}

1 Journals

Édition électronique

URL : http://journals.openedition.org/aspd/354

DOI : 10.4000/aspd.354

ISSN : 1663-9669

Éditeur

Institut de hautes études internationales et du développement

Édition imprimée

Date de publication : 1 octobre 2005

Pagination : 117-125

ISSN : 1660-5934

\section{Référence électronique}

Gilles Carbonnier, «Privatisation de la guerre et sous-traitance des services publics : défis pour l'action humanitaire ", Annuaire suisse de politique de développement [En ligne], 24-2 | 2005, mis en ligne le 19 février 2010, consulté le 07 septembre 2020. URL : http://journals.openedition.org/aspd/354; DOI : https://doi.org/10.4000/aspd.354 


\title{
Privatisation de la guerre et sous-traitance des services publics: défis pour l'action humanitaire
}

\author{
Gilles Carbonnier*
}

\section{Introduction}

La guerre en Irak a mis en exergue le rôle croissant des compagnies militaires privées dans les conflits armés. Les combats à Falloujah impliquant des citoyens américains travaillant pour la firme Blackwater ainsi que la participation des employés de CACI International et de Titan Corp. aux mauvais traitements infligés aux détenus dans la prison d'Abou Ghraïb illustrent la gamme toujours plus large des tâches confiées à ces firmes: formation et entraînement des forces de sécurité, soutien logistique, interrogatoires et services de renseignements, sécurisation de lieux stratégiques, protection rapprochée, participation directe aux hostilités, etc. Une telle privatisation du noyau dur des fonctions de l'Etat soulève de sérieuses questions d'ordre juridique et humanitaire.

En parallèle, les Etats sous-traitent une part croissante des services publics à des contractants privés, par exemple pour assurer l'approvisionnement de la population en eau potable. Ce phénomène de sous-traitance et de privatisation intervient alors que, dans de nombreux pays en développement, les institutions publiques souffrent d'un déficit de légitimité ainsi que de faiblesses structurelles qui les empêchent d'exercer toutes leurs prérogatives et d'opérer sur l'ensemble du territoire national. Dans certaines régions du Nigeria ou d'Indonésie par exemple, les sociétés pétrolières et minières sont devenues les principaux pourvoyeurs de fonds dans des domaines tels que l'éducation, la santé, la sécurité ou encore les infrastructures.

Divers gouvernements, institutions internationales et organisations non gouvernementales (ONG) attendent des sociétés privées qu'elles assument une plus large responsabilité dans la gestion de problèmes globaux tels que la pauvreté, la pénurie d'eau, le réchauffement de la planète ou encore les conflits armés. L'avènement du XXI ${ }^{\mathrm{e}}$ siècle se caractérise ainsi par une profonde remise en question des prérogatives et des responsabilités des institutions publiques face au secteur privé.

* Conseiller économique et coordinateur des relations avec l'économie privée au Comité international de la Croix-Rouge (CICR). L'auteur remercie Emanuela-Chiara Gillard pour ses conseils juridiques sur la question des compagnies militaires privées.

Le texte qui suit est extrait d'un article paru en décembre 2004 dans la Revue internationale de la Croix-Rouge (n 856 , pp. 725-743) sous le titre: «Privatisations, sous-traitance et partenariats publicprivé: Charity.com ou Business.org?». Les opinions qui y sont exprimées sont celles de l'auteur, et ne reflètent pas nécessairement la position du Comité international de la Croix-Rouge. 
Ces évolutions interpellent les organisations humanitaires: les situations de crise tendent à exacerber les problèmes que peuvent susciter la privatisation et la sous-traitance des activités traditionnellement dévolues à l'Etat, avec des conséquences parfois dramatiques pour les populations concernées.

Dans cet article, nous examinons les questions juridiques et humanitaires relatives à l'émergence des compagnies militaires privées avant de nous pencher sur la privatisation et la sous-traitance des services liés à l'approvisionnement en eau, à la gestion des lieux de détention et aux soins de santé.

\section{Privatisation des fonctions militaires}

Phénomène bien connu dès l'Antiquité, et notamment au Moyen-Age, les services de sécurité privés sont à nouveau en plein essor depuis la fin de la guerre froide. Selon les experts, le chiffre d'affaires des compagnies militaires privées dépassait déjà les 100 milliards de dollars en 2002.

Les entreprises privées ont pour premier objectif d'assurer une rentabilité maximale des capitaux investis. Il n'en va pas différemment pour les compagnies militaires privées. Cet objectif de maximisation du profit soulève des questions d'ordre éthique dans le cadre des conflits armés. La compression des coûts peut, par exemple, amener une firme à négliger la formation de son personnel en matière de proportionnalité et de retenue dans l'exercice de la violence. Suivant une rationalité purement économique, certains observateurs font remarquer que ces sociétés pourraient avoir intérêt à voir la demande de leurs services croître par le biais d'une montée du sentiment d'insécurité, voire d'une multiplication des conflits armés.

Pour Peter Singer ${ }^{1}$, expert à la Brookings Institution de Washington, la rationalité économique ne suffit pas à expliquer le recours accru aux firmes militaires privées, qui répondrait davantage à des impératifs politiques. L'auteur indique qu'aux Etats-Unis, les contrats passés par le Pentagone avec des contractants privés de sécurité pour un montant inférieur à 50 millions de dollars ne doivent pas faire l'objet d'une notification au Congrès. De ce fait, le pouvoir législatif risque de perdre une partie du contrôle qu'il est à même d'exercer sur les opérations militaires à l'étranger, en raison des contrats de sous-traitance que le pouvoir exécutif est à même de conclure directement avec les sociétés privées de sécurité.

L'émergence des compagnies militaires privées ainsi que leurs relations avec les Etats posent de nombreuses questions d'ordre juridique. La première porte sur le statut de ces compagnies et de leurs employés dans les conflits armés. L'article 47 du Protocole additionnel aux Conventions de Genève du 12 août 1949 relatif à la protection des victimes des conflits armés internationaux (Protocole I) stipule qu'un mercenaire n'a pas droit au statut de combattant ou de prisonnier de guerre ${ }^{2}$ et précise que le terme «mercenaire» s'entend de toute personne

1 Peter Singer, Corporate Warriors: The Rise of the Private Military Industry. The Privatization of War, Ithaca, Cornell University Press, 2003.

2 Cet article n'interdit pas le mercenariat en tant que tel, contrairement à la Convention de l'Organisation de l'unité africaine (OUA) sur l'élimination du mercenariat en Afrique du 3 juillet 1977 ainsi 
qui remplit six critères cumulatifs. Toutefois, cette acception est si restrictive que, dans la pratique, les dirigeants des compagnies militaires privées devraient être bien imprudents et malchanceux pour que leurs employés tombent dans cette catégorie! Vu qu'un tel cas de figure est plutôt rare dans la pratique, les employés des compagnies militaires privées engagés dans un conflit armé international ont alors le statut de civil ou celui de combattant. Au titre du droit international humanitaire $(\mathrm{DIH})$, ils sont considérés comme des civils à moins qu'ils ne soient de facto intégrés aux forces armées d'une partie au conflit (cf. article 4 de la Convention de Genève relative au traitement des prisonniers de guerre du 12 août 1949; cf. article 43 du Protocole I). Ils perdent toutefois le bénéfice de la protection accordée aux personnes civiles contre les attaques s'ils participent directement aux hostilités, et ce pendant la durée de cette participation (cf. article 51 du Protocole I), tout en conservant leur statut de civil.

Même si le DIH n'offre pas de définition univoque de ce qui constitue une participation directe aux hostilités, il n'en demeure pas moins que certaines activités menées par les compagnies militaires privées tombent clairement sous cette catégorie. Tel est le cas de l'usage de la force armée pour défendre un objectif militaire, du ciblage et du lancement de missiles ou du transport de munitions. Dans ce dernier cas, on peut imaginer que les camionneurs originaires de pays à bas revenu, attirés par les salaires offerts par des entreprises privées pour transporter du matériel militaire dans le contexte d'un conflit armé, ne sont pas forcément conscients qu'ils constituent une cible «légitime» au titre du DIH. Il en va de même pour des contractants privés qui seraient chargés de la sécurité d'objectifs militaires et seraient victimes des «dommages collatéraux» relatifs à une attaque visant l'un de ces objectifs.

Du fait que les employés de compagnies militaires privées sont réputés être des civils tant et aussi longtemps qu'ils ne sont pas intégrés dans les forces armées d'une partie au conflit, il existe un risque réel de voir la multiplication des contractants privés de sécurité sur le terrain renforcer les difficultés relatives à la distinction effective entre civils et combattants dans la conduite des hostilités. La conséquence en serait un affaiblissement de la protection dont bénéficient les personnes civiles dans la pratique.

L'émergence de ces nouveaux acteurs suppose une préoccupation additionnelle pour le Comité international de la Croix-Rouge (CICR): s'assurer que la privatisation d'activités qui étaient traditionnellement exécutées par des armées régulières ou des forces d'opposition - du moins ces deux derniers siècles - n'a pas d'effets négatifs sur les populations civiles touchées par les conflits armés. Aussi est-il essentiel que les Etats assument leur responsabilité de faire respecter le DIH, y compris par les contractants privés de sécurité qui opèrent sur leur territoire ou qu'ils contractent pour des opérations à l'étranger. A ce titre, l'article 1 commun aux Conventions de Genève de 1949 stipule que les Etats signataires s'engagent non seulement à respecter eux-mêmes, mais aussi à faire respecter ces conventions.

qu'à la Convention internationale contre le recrutement, l'utilisation, le financement et l'instruction des mercenaires du 4 décembre 1989. 
Le Projet d'articles sur la responsabilité de l'Etat pour fait internationalement illicite, élaboré par la Commission du droit international des Nations unies et adopté par l'Assemblée générale en 2001, confirme en outre un principe de droit international public. Il énonce que les Etats sont responsables non seulement des actes commis par leurs propres organes, mais aussi de ceux commis par toute personne ou entité qui n'est pas un organe de l'Etat, mais qui est habilitée par le droit de cet Etat à exercer des prérogatives de puissance publique (art. 5). L'article 8 ajoute que «le comportement d'une personne ou d'un groupe de personnes est considéré comme un fait de l'Etat d'après le droit international si cette personne ou ce groupe de personnes, en adoptant ce comportement, agit en fait sur les instructions ou les directives ou sous le contrôle de cet Etat».

Ainsi, les Etats ne peuvent pas se soustraire à leurs obligations au titre du DIH en sous-traitant certaines activités militaires à des sociétés privées. $\mathrm{Si}$, par exemple, un Etat détient des prisonniers de guerre et confie la gestion des lieux de détention à une compagnie privée, il ne lui en incombe pas moins de s'assurer que la gestion de ces lieux répond aux normes fixées dans la troisième Convention de Genève et il ne peut se prévaloir de ce que des manquements graves seraient le seul fait de la compagnie privée. En outre, les Etats qui engagent des compagnies militaires privées sont responsables des violations du DIH commises par ces dernières et par leurs employés.

Les compagnies militaires privées ainsi que leurs employés portent également une responsabilité pour des violations du DIH qu'ils commettraient. Si cette responsabilité est clairement établie, les mécanismes de mise en œuvre qui permettent de traduire une compagnie en justice ou de poursuivre des employés qui auraient commis des crimes laissent par contre à désirer. La responsabilité civile des entreprises privées est un principe généralement bien ancré dans le droit et dans la pratique. Par contre, leur responsabilité pénale est nettement plus limitée. De même, si la responsabilité pénale des auteurs de violations du DIH est clairement reconnue, il peut s'avérer difficile d'engager des poursuites judiciaires à leur encontre dans la pratique. Tant les compagnies que leurs employés bénéficient parfois d'une totale impunité dans les pays où ils opèrent, par exemple lorsque le système judiciaire est incapable de fonctionner en raison du conflit armé. Il peut aussi s'avérer difficile de poursuivre les auteurs de violations du DIH dans le pays où la compagnie militaire privée a son siège social, du fait que les actes répréhensibles ont été commis à l'étranger et que les tribunaux concernés ne peuvent parfois pas exercer de juridiction extraterritoriale ou ne souhaitent pas le faire pour des raisons pratiques (par exemple, faute de preuves) ou politiques. Dès lors, il est impératif de clarifier ces questions pour s'assurer que des sanctions soient prises en cas de violations du DIH, quels que soient la nationalité de l'auteur des abus, celle de la société pour laquelle il travaille et le pays dans lequel la violation a été commise. Il s'agit d'éviter que le flou juridique existant ne confère une immunité de fait aux employés de compagnies militaires privées auteurs de violations du DIH.

La question des droits et responsabilités de tous les acteurs concernés par les activités d'une compagnie militaire privée devient encore plus complexe lorsque cette dernière sous-traite elle-même une partie de son mandat à des milices privées locales, ou qu'elle opère pour le compte d'une entreprise multinationale plutôt que pour un Etat. Trop souvent, les contrats confiés à des compagnies 
militaires privées ne comportent aucune clause relative au respect des droits de l'homme ou du droit international humanitaire. Aussi est-il impératif qu'un cadre normatif adéquat soit adopté par les Etats. En outre, les contrats passés entre les diverses parties ainsi que la formation des employés des sociétés privées doivent concourir à minimiser les risques de violations du DIH, dont pourraient pâtir les communautés touchées par les conflits armés.

\section{Privatisation de l'approvisionnement en eau}

Depuis plusieurs décennies, les régies publiques chargées de l'approvisionnement en eau potable et du traitement des eaux usées sont partiellement privatisées, notamment dans les pays en développement, sous l'impulsion des institutions financières internationales. Ainsi, la gestion des stations de pompage et de traitement de l'eau, de même que le système d'adduction d'eau et la perception des redevances auprès des usagers, ont été confiés à des entreprises multinationales par le biais de contrats de concession ou d'affermage. Par ces mesures, la Banque mondiale et d'autres institutions financières entendent améliorer l'efficacité et la rentabilité du secteur.

Or, le marché de l'eau comporte diverses spécificités qui le distinguent d'autres marchés. La rentabilité du secteur dépend, en particulier, du prix de vente du mètre cube de l'eau, qui est souvent soumis à des plafonds arbitrairement fixés par les gouvernements pour des motifs politiques. En cas de crise humanitaire, l'accès à l'eau potable demeure une priorité absolue pour éviter les épidémies et préserver la santé publique, notamment dans les centres urbains. De ce fait, il est essentiel que les opérateurs privés continuent à fournir leurs services à la population en cas de guerre, même s'il n'est techniquement plus envisageable de prélever les redevances auprès des consommateurs, et par conséquent de générer des profits. Priver la population d'eau potable constitue en outre une violation grave du DIH.

Sur la base d'un simple calcul de rentabilité, ces compagnies sont tentées de se retirer du marché en cas de conflit armé pour éviter les pertes. Au nom de la responsabilité de l'entreprise et dans une perspective de long terme, certaines entreprises décident néanmoins de poursuivre leur travail malgré la situation de guerre. Elles cherchent alors à limiter les pertes par divers moyens, y compris le recours aux fonds disponibles pour l'aide humanitaire et la reconstruction.

Pour les Etats donateurs comme pour les organisations humanitaires, il est essentiel d'assurer l'approvisionnement en eau de la population. La question se pose alors de savoir si on peut - et jusqu'à quel point - soutenir une entreprise multinationale, sans que les fonds et le matériel mis à disposition ne deviennent une subvention indue, contraire aux dispositions de l'Organisation mondiale du commerce et injustifiable envers les contribuables et les concurrents. Une option consiste à avancer les fonds en précisant que ceux-ci seront déduits des dettes de l'Etat envers l'entreprise concessionnaire dès que la perception des redevances auprès des consommateurs aura pu être rétablie. En tout état de cause, en cas de conflit armé, la responsabilité des entreprises dans des secteurs aussi vitaux que l'approvisionnement en eau mérite d'être beaucoup plus clairement définie dans les contrats de concession, notamment lorsque la Banque mondiale ou d'autres organisations internationales soutiennent le processus de privatisation. Une 
option additionnelle serait d'établir un mécanisme international de couverture financière des risques de guerre. Les sociétés privées se verraient contraintes de poursuivre leurs activités dans des domaines aussi vitaux que l'eau même en cas de conflit armé, mais avec l'assurance de limiter les pertes financières par le biais d'une assurance appropriée.

Certains responsables humanitaires refusent de travailler en partenariat avec les sociétés transnationales pour des raisons de principe. D'autres se demandent pourquoi montrer une telle réticence à l'égard des opérateurs privés alors que la communauté humanitaire soutient des régies publiques dans d'autres pays en crise, sans jamais effectuer d'audit détaillé, même si certaines de ces régies sont connues pour leur manque de transparence et la mauvaise gestion des fonds octroyés. Ces diverses positions traduisent des sensibilités personnelles et des positions propres à chaque organisation.

\section{Privatisation du système carcéral}

Dans de nombreux pays, certains établissements pénitentiaires sont gérés en partenariat entre les secteurs public et privé. Des services aux détenus tels que la restauration et les soins de santé sont sous-traités à des sociétés privées. Dans les pays anglo-saxons ${ }^{3}$ notamment, les sociétés privées ${ }^{4}$ en sont venues à gérer certains établissements pénitentiaires dans leur ensemble, y compris en ce qui concerne les services de surveillance et de gardiennage. Il existe une littérature abondante sur ce phénomène et sur ses implications, notamment en matière de respect des droits de l'homme. Il a par exemple été relevé que l'objectif de maximisation du profit poursuivi par les sociétés privées peut entrer en contradiction directe avec les contraintes et objectifs du système carcéral, comme par exemple garantir des conditions de détention satisfaisantes aux personnes internées, ne pas abuser du travail des détenus pour en tirer profit ou encore préparer et favoriser la réintégration sociale des détenus.

Dans le contexte des conflits armés, ce phénomène de privatisation est relativement nouveau et n'a fait que récemment la une des journaux avec les abus commis dans la prison d'Abou Ghraïb en Irak. Lorsque les employés d'une société multinationale commettent des violations du droit international humanitaire à l'encontre de détenus dont ils ont pour mission de s'occuper, diverses questions se posent. Qui est responsable et qui doit être sanctionné? L'employé, la compagnie, le ministère de tutelle, ou tous les trois? Quelle est la responsabilité de l'Etat d'origine de la société privée et de l'Etat où les abus ont été commis? Sous quelle juridiction et selon quelles procédures des sanctions doivent-elles être prises? Le contrat passé entre l'Etat et la compagnie prévoit-il des dispositions spécifiques à cet effet? Le CICR doit-il adresser ses observations suite à ses visites et entretiens avec les détenus aux seuls Etats, ou aussi aux compagnies impliquées dans les interrogatoires des prisonniers? Avec qui doit-il privilégier le dialogue pour améliorer les conditions des détenus? Les réflexions juridiques élaborées plus haut pour les compagnies militaires privées fournissent quelques réponses. Dans ce cas aussi, le CICR insiste sur la responsabilité des Etats, même s'ils ont délégué certaines de leurs prérogatives à des sociétés privées.

3 Australie, Grande-Bretagne, Etats-Unis et Nouvelle-Zélande, de même qu'Afrique du Sud.

4 Par exemple, Group 4 Securicor, Serco, GEO Group Inc. 


\section{Privatisation des services de santé}

L'accès gratuit aux soins n'est pas ou n'est plus une réalité pour les patients dans la grande majorité des pays en crise, à l'exception des soins fournis directement par les organisations humanitaires. Toutefois, les difficultés d'accès aux soins de santé pour les populations vulnérables ne sont pas le lot des seules crises humanitaires. Dans la plupart des pays en développement, on assiste à l'émergence d'un système de santé à double vitesse, avec des structures privées qui fournissent des soins de qualité auxquels seuls les plus riches peuvent accéder, et des structures publiques en faillite qui sont toujours plus nombreuses à introduire un système de recouvrement partiel des coûts par la facturation des soins. Très souvent, les employés mal payés prélèvent des taxes et honoraires informels multiples auprès des patients. Cette privatisation «de l'intérieur», totalement informelle, s'effectue sans la moindre transparence. Dans d'autres cas, les collectivités publiques et les centres de santé ainsi que leurs usagers s'entendent pour établir un système de prépaiement collectif par le biais d'une couverture d'assurance, comme c'est par exemple le cas dans certaines municipalités de Serbie-et-Monténégro.

Les situations de conflit armé ont ceci de spécifique qu'elles exacerbent les besoins de la population et la vulnérabilité des personnes les plus démunies. De plus, le DIH comprend l'obligation de garantir aux victimes d'un conflit armé l'accès aux soins de santé. Or, la privatisation formelle et informelle des soins rend d'autant plus difficile l'action des organisations humanitaires qui, souvent, cherchent à soutenir les structures de santé existantes plutôt qu'à s'y substituer, tout en garantissant l'accès des personnes dont la santé est directement affectée par le conflit armé. Dans les situations de crises aiguës, il arrive que l'accès aux soins ne soit pas garanti aux victimes du conflit - que ce soit les populations déplacées ou les blessés de guerre - parce que le personnel de santé s'occupe en premier lieu des clients solvables, à savoir ceux qui sont capables de fournir une rétribution adéquate, et de la population résidente, mieux intégrée dans les réseaux locaux.

Pour les organisations humanitaires qui s'efforcent de soutenir les structures de santé primaire et secondaire durant les périodes de crise, il devient toujours plus important de comprendre le fonctionnement de ces structures et de fournir des conseils en matière de gestion administrative et financière, pour être mieux à même d'apporter une aide pertinente et durable. L'expérience montre que, même si les humanitaires interviennent dans l'urgence, leur travail de soutien aux systèmes de santé locaux tend à se prolonger de nombreuses années. Une intervention dans la durée requiert des compétences toujours plus vastes eu égard aux spécificités du «marché» de la santé et des systèmes d'assurance (par exemple privatisation partielle, avec ou sans mécanisme d'assurance, asymétrie d'information, risque moral, sélection adverse, etc.). 
Les crises humanitaires - de par leurs spécificités et les implications dramatiques pour les communautés affectées - mettent en exergue de manière particulièrement saillante les questions de fond que soulèvent la privatisation et la sous-traitance des services publics de base.

La première question tourne autour de la compatibilité entre l'objectif de maximisation du profit, propre aux entreprises privées, et celui du maintien de la sécurité et de la santé publique, propre aux Etats. En effet, le marché en tant qu'institution permet au jeu de l'offre et de la demande ainsi qu'à la concurrence de s'exprimer librement, avec pour objectif d'obtenir une allocation optimale des ressources et de stimuler la créativité et l'innovation pour répondre aux besoins des consommateurs, voire susciter de nouveaux besoins. Or, le marché n'est évidemment doté d'aucune éthique intrinsèque: les mécanismes de l'offre et de la demande s'appliquent aussi bien au marché des actions ou du café qu'au marché de la prostitution infantile ou de la cocaïne. Il appartient à l'Etat de proscrire ou d'encadrer certains marchés pour des raisons d'ordre public, de sécurité, de morale, de santé ou autres. Il lui appartient aussi de résister aux pressions de sociétés privées qui pourraient être tentées d'inciter les autorités à stimuler la demande de leurs services, à savoir à engager, poursuivre ou intensifier un conflit armé dans le cas de compagnies militaires privées.

Il est en outre essentiel que les contrats de sous-traitance confiés par des gouvernements à des sociétés privées dans des domaines aussi sensibles que l'approvisionnement en eau et la gestion des prisons prévoient des clauses précises relatives aux responsabilités et obligations de chaque partie en cas de force majeure (un conflit armé par exemple), ainsi que des mécanismes d'incitation économique et financière pour garantir que les préoccupations humanitaires soient prises en compte de façon prioritaire. Sans quoi, les sociétés privées auront beau jeu de se référer aux termes des contrats passés avec les autorités, contrats qui ne comporteraient aucune disposition à cet effet.

La deuxième question porte sur le respect du droit international par les sociétés privées, et sur la responsabilité de l'Etat lorsque les activités de ces sociétés sont susceptibles de mettre en danger le respect du droit international humanitaire. Comme nous l'avons vu, les Etats sont responsables des actes commis par toute personne ou entité habilitée à exercer des prérogatives de puissance publique ou qui agit (de facto) sur les instructions ou sous le contrôle de l'Etat. Cependant, le risque de voir des Etats se soustraire à leurs obligations au titre du droit international par le biais de la privatisation et de la sous-traitance est bien réel et requiert une attention accrue.

La troisième question porte sur la rationalité économique de ce phénomène, principal argument des responsables politiques qui encouragent les privatisations en invoquant les économies qui en résultent. Or, le bilan de la privatisation des services publics reste sujet à controverses. Une étude publiée en 1998 par Abt Associates ${ }^{5}$ - une société de conseil nord-américaine qui fournit des

5 «Private Prisons in the United States: An Assessment of Current Practice», Cambridge (Massachusetts), Abt Associates Inc., 16/07/1998, p. 56, cité in The Sentencing Project, Prison Privatization and the Use of Incarceration, Washington, D.C., mise à jour de septembre 2004, <www.sentencingproject. org/pdfs/1053.pdf>. 
conseils dans le domaine des réformes économiques et de la privatisation, et ne peut être soupçonnée de véhiculer des préjugés négatifs à cet égard - conclut que les analyses et données disponibles ne démontrent pas que le recours à des sociétés privées dans le domaine pénitentiaire permet de réaliser des économies. Plus incisive, l'organisation War on Want affirme que la privatisation de nombreux services publics a aggravé la pauvreté dans les pays en développement et que l'agence britannique de coopération au développement, en soutenant ces réformes, a contribué à enrichir les consultants et les entreprises privées britanniques plutôt qu'à réduire la pauvreté dans les pays bénéficiaires ${ }^{6}$. Les arguments relatifs aux avantages économiques de différents arrangements institutionnels entre les secteurs public et privé doivent par conséquent faire l'objet d'un examen rigoureux, et doivent être pondérés eu égard aux considérations d'ordre politique, social et autres.

Les organisations humanitaires ont intérêt à se doter d'une meilleure compréhension des apports et du mode de fonctionnement des opérateurs privés. Ce faisant, elles amélioreraient leurs capacités à traiter avec ces nouveaux intervenants dans les crises humanitaires, ce dont profiteraient les communautés visées. Toutefois, de tels échanges entre organisations humanitaires et sociétés privées ne sauraient en aucun cas dédouaner les Etats de leurs obligations au titre du droit international, notamment dans des secteurs «en voie de privatisation» tels que la formation des forces armées, la gestion de lieux de détention ou la fourniture d'eau potable et de soins médicaux.

6 Duncan Campbell, «UK Accused of Using Aid to Promote Privatisation», The Guardian, 27/09/2004. 